\title{
Ambulatory accelerometry to quantify involuntary movements and tics in the syndrome of Gilles de la Tourette
}

\author{
JOKE H. M. TULEN, WIM H. GROENEVELD, JUDITH H. G. J. K. ROMERS, \\ SANDER J. A. DE VRIES, and BEN J. M. VAN DE WETERING \\ University Hospital Rotterdam-Dijkzigt, Rotterdam, The Netherlands \\ and Erasmus University, Rotterdam, The Netherlands
}

\begin{abstract}
Gilles de la Tourette syndrome is a chronic neuropsychiatric disorder characterized by recurrent and involuntary tics, in addition to complex behavioral symptoms. Objective quantification of the nonspecific movements in Tourette patients can contribute much to understanding the pathophysiology of this disease. We used three accelerometers to characterize head movement patterns and to objectively quantify head motility in the lateral, sagittal, and transversal planes in 9 Tourette patients and 14 controls during periods of rest, conversation, and watching a videotape with an entertaining program. Characteristic head movement patterns can be documented by means of accelerometry. Head motility levels in the lateral, sagittal, and transversal planes were significantly higher in the patients than in the controls during all the procedures. The patients and the controls showed a similar significant increase in head motility during conversation, but not during video watching. This first study shows that for both standardized and ambulatory research, accelerometry may provide an objective tool by which to quantify the severity and temporal dynamics of tics or nonspecific movements.
\end{abstract}

The typical characteristics of the syndrome of Gilles de la Tourette (GTS) consist of recurrent and involuntary motor and vocal tics, which are frequently accompanied by behaviors related to attention-deficit hyperactivity disorder or obsessive compulsive disorder (see, e.g. Knell \& Comings, 1993; Pauls \& van de Wetering, 1995; Shapiro, Shapiro, Young, \& Feinberg, 1988). Increased eye blinking, facial twitches, and neck jerking are considered to be simple motor tics, whereas simple vocal tics are such sounds as throat clearing, squealing, and grunting. Complex motor tics include rubbing, touching, dystonic postures, and symmetry behaviors; yelling (obscene) phrases is an example of a complex vocal tic. The tics usually occur in a short and explosive manner, show a waxing and waning course over time, and are often exacerbated by stress and anxiety (Leckman, Walker, Goodman, Pauls, \& Cohen, 1993). The patient can also temporarily suppress tics-for instance, during performance of an attentiondemanding task - but suppression may be followed by a rebound increase in the frequency and intensity of the tics.

Objective quantification of the frequency and intensity of tics in patients with GTS is essential for diagnostic purposes, for evaluation of therapy efficacy, and to increase our understanding of the pathophysiology of the

Correspondence concerning this article should be addressed to J. H. M. Tulen, University Hospital Rotterdam-Dijkzigt, Department of Psychiatry, Dr. Molewaterplein 40, 3015 GD Rotterdam, The Netherlands (e-mail: tulen@psy.fgg.eur.nl). disease. The difficulty of developing reliable methods for the objective assessment of tic severity primarily relates to the variable presentation of tic symptoms, comprising aspects of body localization, frequency, intensity, complexity, and noticeability, in addition to time- and situation-specific variations within and across subjects (Kurlan \& McDermott, 1992; Peterson \& Leckman, 1998; Shapiro et al., 1988). Furthermore, the tics and abnormal movements need to be interpreted in relation to the normal background behavior. At present, several clinicianbased rating scales exist to assess the multiple clinical features that contribute to the overall severity of Tourette's disorder (e.g., see Leckman et al., 1989; Shapiro et al., 1988), whereas videotape-based tic countings have been used to obtain a more quantitative index of tic severity and the temporal dynamics of tics (e.g., Chappell et al., 1994; Goetz, Tanner, Wilson, \& Shannon, 1987; Peterson \& Leckman, 1998). Both approaches show shortcomings regarding the objectivity and reliability of the data obtained, as well as the fact that data collection is restricted to controlled situations (during which video recordings and/or interviews can be made).

Accelerometry (in combination with the use of an ambulatory digital recorder) may be the appropriate alternative for objectively quantifying both involuntary movements and motor tics in controlled and ambulatory situations in Tourette patients. With accelerometers, both the angle of the sensor relative to the gravitational axis and the actual acceleration of the sensor can be monitored (Veltink, Bussmann, de Vries, Martens, \& van Lummel, 
1996). Previous research has shown that body-mounted accelerometers can be used successfully to objectively quantify aspects of physical activities, such as body postures (lying, sitting, standing) and locomotor activities (walking, cycling, etc.), in healthy subjects and several clinical populations (e.g., amputees, patients after failed back surgery, migraine patients; Bussmann, Tulen, van Herel, \& Stam, 1998; Bussmann, van de Laar, Neeleman, \& Stam, 1998; Tulen, Bussmann, van Steenis, Pepplinkhuizen, \& Man in 't Veld, 1997; Tulen, Stronks, Bussmann, Pepplinkhuizen, \& Passchier, 2000). In this study, we quantified involuntary head movements and head tics by means of accelerometry in a standardized situation during which Tourette patients and control subjects performed two tasks (conversation, watching a videotape). By using three accelerometers, the position of the head in the lateral, sagittal, and transversal planes and the actual acceleration of the head during normal and abnormal movements in the three planes could be documented. Qualitatively, we scrutinized the signals in comparison with simultaneously recorded videotapes in order to define the characteristic patterns of head movements/tics in the accelerometer signals. Quantitatively, we analyzed the accelerometer signals by computing an overall head motility index for the three planes in order to study baseline differences and task-specific effects regarding head movements between patients and controls. As such, this study is the first to objectively quantify task-related effects on involuntary movements in patients with GTS by means of a method that can be used in both controlled and ambulatory situations.

\section{METHOD}

\section{Subjects}

Patients. Nine patients ( 7 males, 2 females; mean age, 37.6 years; $S D, 13$; age range, 20-56 years) with current symptoms of Tourette syndrome participated in a study aimed at the objective quantification of eye blinks, tics, and head movements. The patients were recruited within a period of 2 months from the psychiatric outpatient service of the University Hospital Rotterdam-Dijkzigt and were diagnosed by a senior psychiatrist according to the criteria of the DSM-IV (American Psychiatric Association, 1994). All the patients suffered from multiple and single motor and vocal tics, as well as obsessive- compulsive behaviors, had variable durations of the illness, and, at the time of the intake, scored mild to moderate on the severity of the syndrome according to the Yale Global Tic Severity Scale (Leckman et al., 1989). Five of the 9 patients were on neuroleptics at the time of the study; 1 patient received an antidepressant.

Controls. The control group consisted of 14 healthy volunteers ( 7 females, 7 males; mean age, 31.8 years; $S D, 14.3$ ), who were unrelated to the patients. The subjects had no personal history of movement disorders and were free of psychoactive drugs at the time of the study.

During the intake, both the patients and the controls were informed about the procedures, measurements, and video recordings and gave written informed consent to participate in the study.

\section{Procedure}

Each subject participated in a recording session of about $1 \mathrm{~h}$ at the Psychophysiological Laboratory of the University Hospital RotterdamDijkzigt. During the entire procedure, the subject was not allowed to smoke or drink coffee. The subject sat in a comfortable chair while recordings of head movements were made by means of accelerometers; a videocamera (Panasonic NV-S7E), placed at a distance of about $2 \mathrm{~m}$ from the subject, recorded in detail the movements of the face, head, and shoulders of the subject. The following sequence of tasks was performed: (1) rest1, a 10-min period of rest; (2) conversation, an interview/conversation of 5 min during which the subject talked freely with the researcher about his/her favorite hobbies; (3) rest2, a 5-min period of rest; and (4) video, watching a videotape (of about $15 \mathrm{~min}$ ) on a television monitor, showing an entertaining television program, no task being required from the subject, the instruction being merely to relax and watch the video. During rest 1 and rest 2 , the subjects were asked to relax, to remain seated, and not to speak to the researcher (who was present in the room during the whole procedure). At the end of the session, the GTS patients were asked whether they felt that they had suppressed their tics during the measurements.

\section{Measurements and Analyses}

Measurement of the head movements was performed by means of three uniaxial 5-g piezo-resistive accelerometers (IC-sensors 3031) that were attached to an elastic band, placed above the eyes of the subject. Accelerometers provide signals that are a mixture of two components: the gravitational force (absolute angle information) and the actual acceleration of the sensor (Veltink et al., 1996). Sensor 1, placed above the left ear, recorded the position and movements of the head in the lateral plane (i.e., toward the left or right shoulder); Sensor 2, placed on the forehead, recorded the position and movements of the head in the sagittal plane (i.e., forward or backward); and Sensor 3, also placed on the forehead, recorded the position and movements of the head in the transversal plane (i.e., rotation of the head to the left or to the right). Because of the oval/round characteristics of the head, the sensors could not be placed entirely perpendicular to the direction of the gravitational field; some cross-talk between the three planes, as reflected in the accelerometer signals, therefore could occur. For practical purposes, we allowed this (but controlled for this in our analyses procedures), since the aim of our recording method is to quantify movements in ambulatory situations in the habitual environment of the subject. The accelerometer signals were stored at a sample frequency of $64 \mathrm{~Hz}$ on a digital portable recorder. The datafiles were uploaded to a personal computer and were processed and analyzed with the WinDaq/-EX waveform browser (Version 1.38; Dataq Instruments) and custom software modules (written in LabVIEW 5.1, National Instruments). Our quantif ication was based on the signals of Sensor 1 (lateral), Sensor 2 (sagittal), and the difference signal of the signals of Sensor 3 and Sensor 1 (transversal plane), in order to compensate for the sensitivity of Sensor 3 in the lateral plane (for an example of head movements in the three planes, see Figure 1A).

Qualitative analyses. A clinical expert (B.J.M.v.d.W.) and an independent researcher compared the videotapes with the corresponding accelerometer signals in a search for specific patterns and types of movements/tics in the accelerometer signals. As a first approach, our focus was on the identification of unique signal features for the Tourette patients, corresponding with specific head tics; these qualitative analyses were not performed blind with respect to patients versus controls.

Quantitative analyses. The accelerometer signals of the first 4 min of each baseline or task period were quantitatively analyzed. A head motility index was computed for each plane (lateral, sagittal, transversal) in order to obtain a general motility measure, reflecting changes in both frequency and intensity of movements. Each of the three signals was high-pass filtered (4th order Butterworth filter, $0.5 \mathrm{~Hz}$ ) and rectified to suppress the static components in the signal caused by the positions of the head; the resulting signals were integrated over 1-min intervals. Per baseline and task period, the data of the 4 min were averaged. 

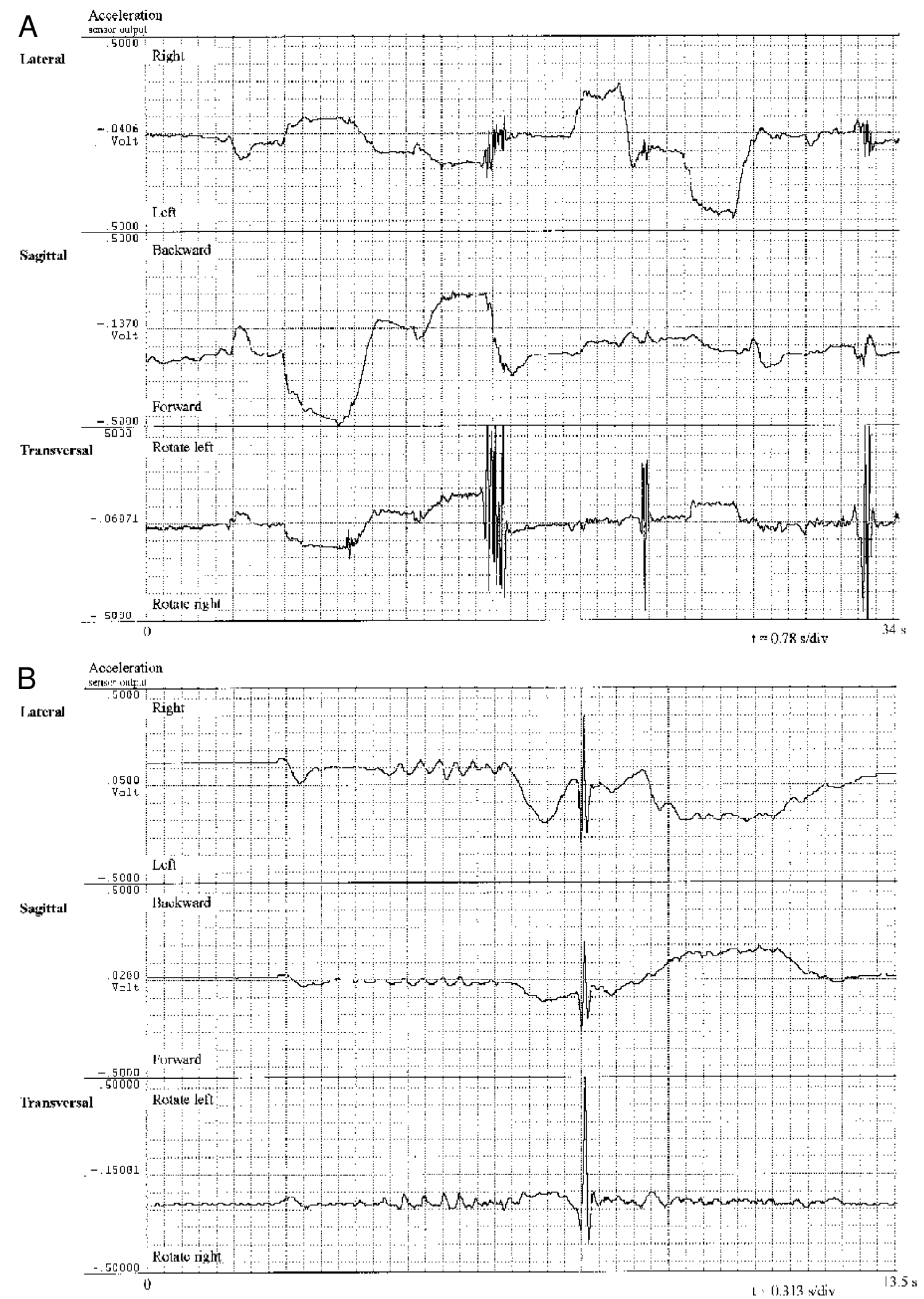

Figure 1. Accelerometer signals reflecting lateral, sagittal, and transversal head movements. (A) Calibration movements and head tics of a Tourette patient. (B) Example of an oscillatory movement pattern and an isolated fast head movement during conversation of a Tourette patient. 


\section{Statistics}

Differences between the patients and the controls regarding head motility in the three planes were evaluated by means of MannWhitney $U$ tests. Within-group analyses were performed by means of Wilcoxon tests. A $p$ value of $<.05$ was used to indicate a significant difference.

\section{RESULTS}

\section{Head Movement Patterns: Oscillations and Fast Movements}

Two typical head movement patterns were recognized in the accelerometer signals.

1. Oscillatory head movements (about $3-4$ cycles per second, varying in length between 1 and $4 \mathrm{sec}$; for an example, see Figure 1B), occurred in both the patients and the controls, primarily in the transversal and lateral planes, as if to shake one's head; these oscillations were apparent during the conversation task when the subjects were speaking. The pattern was distinct from nodding, which occurred in response to specific questions from the researcher (affirmation or denial).

2. Fast head movements always corresponded to isolated or complex bursts of motor tics in the Tourette patients (head jerking; for examples, see Figure 1A and 1B). Isolated fast motor tics (Figure 1B) were characterized by one sharp peak, not related or influenced by other head movements, the total duration of the fast movement always being less than $0.22 \mathrm{sec}$, but with varying appearance in amplitude and direction of movement (lateral, sagittal, transversal). Complex forms of fast head movements and tics could also be identified in the accelerometer signals; these were characterized by multiple peaks, with variations in frequency, amplitude, or direction of movement. The fast head tics can be differentially present in the lateral, sagittal, or transversal plane, as is illustrated by the accelerometer signals presented in Figure 1A.

\section{Head Motility}

Conversation induced a significant increase in head motility in both the controls and the patients, in all three movement planes (Table 1, Figure 2). Video watching did not significantly affect head motility in the controls and patients. The patients showed higher head motility levels than did the controls during baseline and task periods, in the lateral, sagittal, and transversal planes (Table 1 ; all periods, $p<.05$ ). Head motility levels in the three planes differed significantly in the control group (Friedman analyses for all periods, $p<.01$ ), with motility in the sagittal plane showing the lowest levels. In the patient group, there was no significant difference in head motility in the three planes.

\section{DISCUSSION}

Accelerometry was explored as a novel method to objectively quantify aspects of overt motor behavioral patterns in Tourette patients-specifically, head movements. Fast head movements with characteristic signal features could be recognized in the accelerometer signals, corresponding to isolated fast motor tics or bursts of fast tics in the patients. Signal patterns corresponding to slower (dystonic or tonic) tics could not be uniquely defined in this first approach; these patterns are more difficult to characterize, because they cannot be unequivocally distinguished from movements that healthy subjects sometimes make.

As a first quantitative approach, we computed a head motility index, an overall movement index reflecting frequency and intensity of head movements above $0.5 \mathrm{~Hz}$. This head motility index during periods of rest was significantly higher in the Tourette patients than in the controls. This suggests a chronic hyperactivity in the patients, comprising both increased nonspecific head movements and head tics. Motility levels based on lateral, sagittal, or transversal head movements of the patients were similar, whereas sagittal head motility levels were lowest in the controls. Apparently, spontaneous head movements in the healthy controls were more frequently (or more intensely) made in the lateral and transversal planes, whereas in the Tourette patients no differentiation was observed.

Table 1

Mean Head Motility (int/min) During Rest and Task Periods, Based on Lateral, Sagittal, and Transversal Head Movements, for Controls and Tourette Patients Separately

\begin{tabular}{|c|c|c|c|c|c|c|c|}
\hline \multirow[b]{2}{*}{ Movement } & \multirow[b]{2}{*}{ Task } & \multicolumn{2}{|c|}{ Controls } & \multicolumn{2}{|c|}{ Patients } & \multicolumn{2}{|c|}{ Mann-Whitney Test } \\
\hline & & $M$ & $S D$ & $M$ & $S D$ & $Z$ & $p$ Values \\
\hline \multirow[t]{4}{*}{ Lateral } & rest 1 & 138 & 57 & 389 & 286 & -2.6 & .008 \\
\hline & conversation & $515 \dagger$ & 184 & $761^{*}$ & 324 & -2.1 & .04 \\
\hline & rest 2 & 180 & 112 & 465 & 244 & -3.3 & $<.001$ \\
\hline & video & 174 & 83 & 359 & 285 & -2.1 & .03 \\
\hline \multirow[t]{4}{*}{ Sagittal } & rest 1 & 109 & 49 & 339 & 166 & -3.4 & $<.001$ \\
\hline & conversation & $375 \dagger$ & 106 & $597 *$ & 249 & -2.3 & .02 \\
\hline & rest2 & 138 & 58 & 422 & 177 & -3.5 & $<.001$ \\
\hline & video & 14 & 81 & 339 & 195 & -2.6 & .008 \\
\hline \multirow[t]{4}{*}{ Transversal } & rest 1 & 171 & 38 & 354 & 175 & -3.0 & .002 \\
\hline & conversation & $429 \dagger$ & 174 & $624^{*}$ & 259 & -2.1 & .03 \\
\hline & rest 2 & 180 & 48 & 432 & 187 & -3.7 & $<.001$ \\
\hline & video & 182 & 49 & 387 & 230 & -2.9 & .003 \\
\hline
\end{tabular}

$* p<.01 . \dagger p<.001$ (Wilcoxon tests, within-group analyses: significant increases from rest 1 to conversation. 

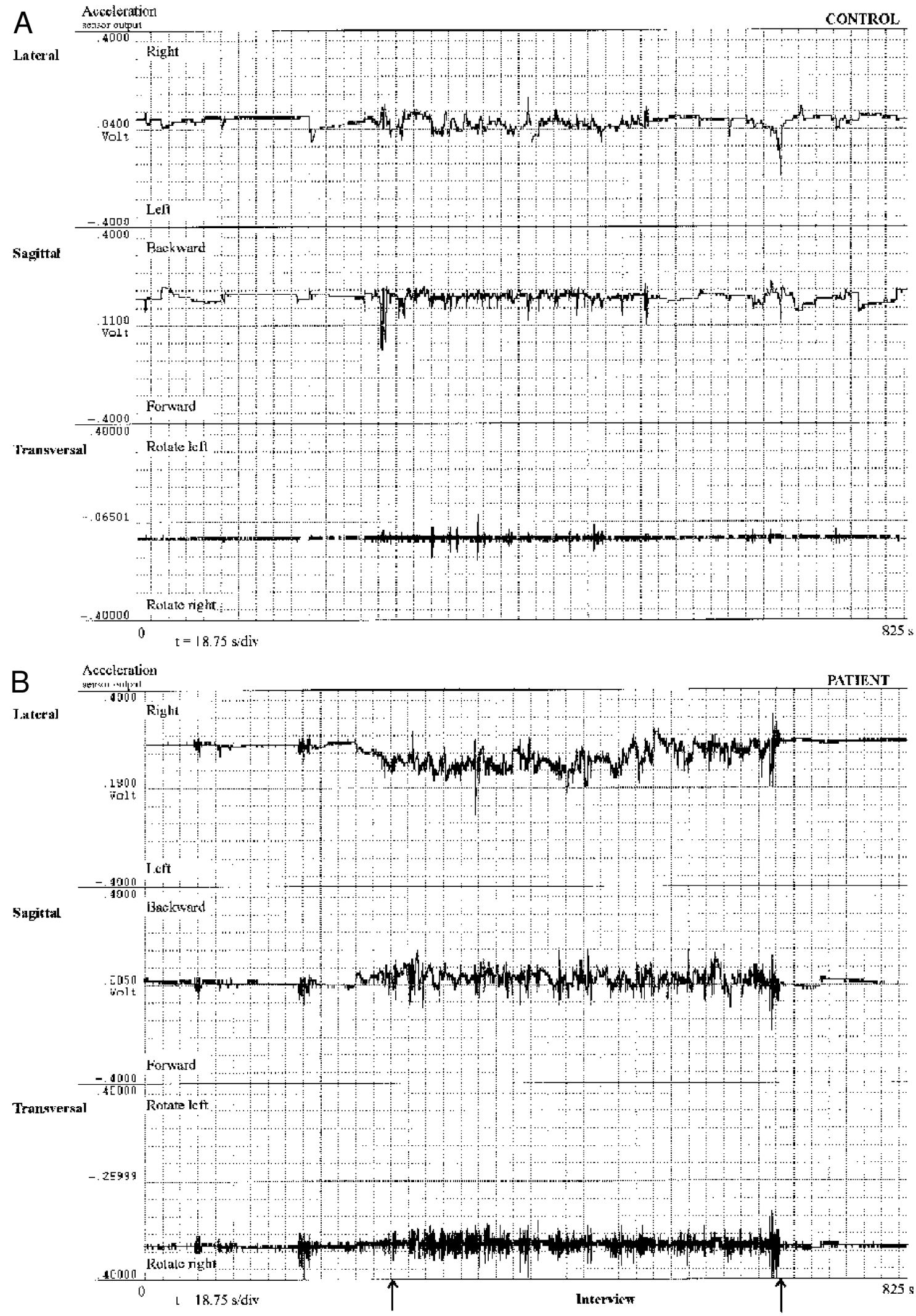

Figure 2. Condensed recording of accelerometer signals reflecting lateral, sagittal, and transversal head movements, illustrating the effect of conversation on spontaneous head movements for a control subject (A) and a Tourette patient (B). The arrows mark the beginning and the end of the conversation period. 
Social interaction, such as occurs during conversation, proved to be an interesting trigger for head movements in the patients and controls. The patients did not differ from the controls in the pattern of their head oscillations, which were primarily made in the lateral and transversal planes when they were speaking to another person. This increase of head movements was also reflected in the head motility index. Head motility levels during the conversation task were higher in the patients than in the controls, reflecting the presence of head tics and other nonspecific movements in the patients. The aim of our method was not to detect the meaning or function of head movements in relation to other nonverbal behavior during communicative interaction, as had been the focus of Altorfer et al. (2000) with the use of ultrasonic transducers. We used conversation as a provoking task to trigger eye blinks (Tulen et al., 1999) and nonspecific head movements and to describe the differences between the Tourette patients and the controls during this task. Our recording method does, however, allow more detailed analyses of communicative interaction and nonverbal behavior, which may be realized in the near future. Watching a videotape did not significantly affect head motility levels in the controls and patients, although motility levels were significantly higher in the patients than in the controls. Previously, we had observed that watching a videotape with an entertaining program resulted in a significant increase in eye tics in the Tourette patients (Tulen et al., 1999). With the approach of the present analyses, we did not establish whether video watching also leads to an increase in fast head movements or tics in the patients.

Because of its exploratory nature, our study contains several limitations. (1) We used a relatively small sample size of patients, a number of whom were on medication. Larger studies with drug-free and treated patients need to be performed in order to relate quantitative indices regarding motility and tics to subjective and clinical estimates of severity of the syndrome in order to unravel similarities and differences between various objective and subjective parameters. Furthermore, relating the objective findings to the efficacy of different types of treatment may contribute to our understanding of the pathophysiology of GTS. (2) Presently, we have no quantitative data on the effects of age or gender on our parameters. However, our methodology can be used to study nonspecific movements and tics in various age groups under controlled and ambulatory conditions.

\section{Future Developments}

Feature extraction and automatic signal processing. 1. Implementation of the signal features of fast head movements and tics in automatic signal-processing procedures may facilitate the quantification of the severity and temporal dynamics of the tics. It may be necessary to optimize sample rate settings (presently, $64 \mathrm{~Hz}$ ) in order to describe the peak acceleration of the very fast tics accurately. Further controlled studies are required in which computerized output needs to be validated against visually analyzed videotapes. Once the validity of the automatic procedures has been established, it is possible to move from controlled to ambulatory studies.

2. Additional research is required to define the unique features of other simple tics and dystonic or tonic tics. This will be a challenging task, owing to the variable presentation of tic symptoms (i.e., unique features may reflect aspects of localization, frequency, intensity, and complexity). Furthermore, some of these movement patterns are difficult to differentiate from normal spontaneous head movements. Although at present, we have focused our attention on movements above $0.5 \mathrm{~Hz}$, quantification of the slower movements may provide an interesting opportunity to look for specific movement distinctions between GTS patients and controls.

3. Spectral analyses of the accelerometer signals (for instance, by means of joint time-frequency analysis) may be explored for its potential for reflecting the intensity and periodicity of involuntary movements and tics.

Instrumentation. 1 . The sensors could not be placed entirely perpendicular to the direction of the gravitational field because of the oval/round characteristics of the head. Optimalization of sensor position is something that also requires attention in the future when other types of tics are studied and when recordings are made under ambulatory conditions.

2. On the basis of validity studies, it might be possible to establish a criterion acceleration value that triggers data collection, in order to reduce the hardware demand for data storage space of the ambulatory system.

3. Simultaneous recording of accelerometer signals with physiological signals (ECG, EMG, EOG) or sound signals is an interesting option for unraveling relationships among motor and vocal tics, muscle tension, and functioning of the autonomic nervous system. With the present digital recorder (Vitaport2), it is feasible to perform this.

\section{Conclusion}

Obtaining reliable methods for the objective assessment of tic severity and (ab)normal nonspecific movements is a difficult task, owing to the variable presentation of tic symptoms and the time- and situation-specific variations within and across subjects. With this first study, we have shown the potential of accelerometry to provide objective quantitative information on spontaneous movements in Tourette patients. In both standardized and ambulatory settings, accelerometry may contribute to an accurate description of the severity and temporal dynamics of tics or nonspecific movements.

\section{REFERENCES}

Altorfer, A., Jossen, S., Wurmle, O. Kasermann, M.-L., Foppa, K \& Zimmermann, H. (2000). Measurement and meaning of head movements in everyday face-to-face communicative interaction. $\mathrm{Be}$ havior Research Methods, Instruments, \& Computers, 32, 17-32. American Psychiatric Association (1994). Diagnostic and statistical manual of mental disorders (4th ed.). Washington, DC: American Psychiatric Press. 
Bussmann, J. B. J., Tulen, J. H. M., van Herel, E. C. G., \& Stam, H. J. (1998). Quantification of physical activities by means of ambulatory accelerometry: A validation study. Psychophysiology, 35, 488-496.

Bussmann, J. B. J., van de Laar, Y. M., Neeleman, M. P., \& Stam, H. J. (1998). Ambulatory accelerometry to quantify motor behaviour in patients after failed back surgery: A validation study. Pain, 74, 153161.

Chappell, P. B., McSwiggan-Hardin, M. T., Scahill, L., RubenStein, M., Walker, D. E., Cohen, D. J., \& Leckman, J. F. (1994). Videotape tic counts in the assessment of Tourette's syndrome: Stability, reliability, and validity. Journal of the American Academy of Child \& Adolescent Psychiatry, 33, 386-393.

Goetz, C. G., Tanner, C. M., Wilson, R. S., \& Shannon, K. M. (1987). A rating scale for Gilles de la Tourette's syndrome: Description, reliability, and validity data. Neurology, 37, 1542-1544.

Knell, E. R., \& Comings, D. E. (1993). Tourette's syndrome and attention-deficit hyperactivity disorder: Evidence for a genetic relationship. Journal of Clinical Psychiatry, 54, 331-337.

Kurlan, R., \& McDermott, M. P. (1992). Rating tic severity. In R. Kurlan (Ed.), Handbook of Tourette's syndrome and related tic and behavioral disorders (pp. 199-220). New York: Marcel Dekker.

Leckman, J. F., Riddle, M. A., Hardin, M. T., Ort, S. I., Swartz, K. L., Stevenson, J., \& Cohen, D. J. (1989). The Yale Global Tic Severity Scale: Initial testing of a clinician-rated scale of tic severity. Journal of the American Academy of Child \& Adolescent Psychiatry, 28, 566-573.

Leckman, J. F., Walker, D. E., Goodman, W. K., Pauls, D. L., \& Cohen, D. J. (1993). Premonitory urges in Tourette's syndrome. American Journal of Psychiatry, 150, 98-102.
Pauls, D. L., \& VAn de Wetering, B. J. M. (1995). The genetics of tics and related disorders. In M. M. Robertson \& V. Eapen (Eds.), Movement and allied disorders (pp. 13-29). New York: Wiley.

Peterson, B. S., \& Leckman, J. F. (1998). The temporal dynamics of tics in Gilles de la Tourette syndrome. Biological Psychiatry, 44, 1337-1348.

Shapiro, A. K., Shapiro, E. S., Young, J. G., \& Feinberg, T. E. (1988). Gilles de la Tourette syndrome. New York: Raven Press.

Tulen, J. H. M., Azzolini, M., de VRies, J. A., Groeneveld, W. H., Passchier, J., \& VAN de Wetering, B. J. M. (1999). Quantitative study of spontaneous eye blinks and eye tics in Gilles de la Tourette's syndrome. Journal of Neurology, Neurosurgery \& Psychiatry, 67, 800-802.

Tulen, J. H. M., Bussmann, J. B. J., van Steenis, H. G., PepplinkHUIZEN, L., \& MAN IN 'T VELD, A. J. (1997). A novel tool to quantify physical activities: Ambulatory accelerometry in psychopharmacology. Journal of Clinical Psychopharmacology, 17, 202-207.

Tulen, J. H. M., Stronks, D. L., Bussmann, J. B. J., Pepplinkhuizen, L., \& Passchier, J. (2000). Towards an objective quantitative assessment of daily functioning in migraine: A feasibility study. Pain, 86, 139-149.

Veltink, P. H., Bussmann, J. B. J., de Vries W., Martens, W. L. J., \& VAN LUMmel R. C. (1996). Detection of static and dynamic activities using uniaxial accelerometers. IEEE Rehabilitation Engineering, $\mathbf{4}$, 375-385.

(Manuscript received October 4, 2000; revision accepted for publication June 2, 2001.) 